\title{
Controlling the Morphology and Mesostructural Orderness of the Mesoporous Silica Nanoparticles
}

\author{
Man-Chien Chao, Hong-Ping Lin, ${ }^{* \dagger}$ and Chung-Yuan Mou \\ Department of Chemistry, National Taiwan University, Taipei, Taiwan 106 \\ ${ }^{\dagger}$ Department of Chemistry, National Cheng Kung University, Tainan, Taiwan 701
}

(Received February 18, 2004; CL-040185)

Using an acid-hydrolyzed tetraethyl orthosilicate in the presence of surfactant and a fast self-assembling reaction at desired $\mathrm{pH}$ value (9.0-5.0) could obtain mesoporous silica nanorods or nanoparticulates.

Recently, mesoporous silica nanoparticles have received much attention because their high connectivity and accessibility have been considered as an advanced support in the catalytic application. ${ }^{1}$ Although the synthetic method and special chemical components have been previously reported to synthesize the mesoporous silica nanoparticles, a theoretical study on the control of the morphology and mesostructure of the mesoporous silica nanoparticles has not been published yet. ${ }^{2-6}$ Herein, we proposed a convenient method based on the concepts of silica chemistry, ${ }^{7}$ surfactant theory and self-assembling kinetics ${ }^{8}$ to prepare the mesoporous silica nanorods and nanoparticulates with ordered and disordered mesostructure using the cationic quaternary ammonium surfactant as mesostructure-directing agent.

Basically, the mesoporous silica nanorods and nanoparticulates were synthesized via an acid-hydrolysis of the silica alkoxides in the presence of surfactant and a fast self-assembling reaction at desired $\mathrm{pH}$ value of 5.0-9.0. In a typical preparation, $1.16 \mathrm{~g}$ of $\mathrm{C}_{16} \mathrm{TMAB}$ (Acrôs) was dissolved into $58.0 \mathrm{~g}$ of water and combined with $5.0 \mathrm{~g}$ of 65 wt $\% \mathrm{HNO}_{3}$ (Acrôs) and $3.0 \mathrm{~g}$ of tetraethyl orthosilicate (TEOS, Acrôs). After stirring for 6$10 \mathrm{~min}$, this clear solution was fast poured into $600 \mathrm{~mL}$ of $0.25 \mathrm{M}$ ammonia solution $(\mathrm{pH}=9.0-10.0)$ or an aqueous solution of $8.12 \mathrm{~g}$ of $\mathrm{CH}_{3} \mathrm{COONa}$ and $1.0-2.0 \mathrm{~g}$ of $\mathrm{NaOH}(\mathrm{pH}=$ 5.0-7.0), and then the white precipitate was formed within seconds. It should be mentioned that the most suitable hydrolysis time in acidic solution is about $6-10 \mathrm{~min}$. A longer hydrolysis time causes a high-degree silica condensation and white precipitate is formed. However, with a shorter hydrolysis time, the silica-hydrolysis extent is not enough, the unhydrolyzed TEOS would bring about a slow silica condensation and the submicron particulates $(>100 \mathrm{~nm})$ are formed instead of nanorods or nanoparticles. The solid product was recovered, washed, and air-dried at room temperature. Yields are typically $>90 \%$ based on silica. The surfactant was removed by calcination at $560{ }^{\circ} \mathrm{C}$ for $6 \mathrm{~h}$ in air.

Figure 1 shows the SEM and TEM microscopic images of the mesoporous silicas prepared in the buffer solutions at different $\mathrm{pH}$ value. At final $\mathrm{pH}$ value of 9.0, the morphology of the mesoporous silica is homogeneously in a rod-like form (Figure 1A). Obviously, the diameter of the nanorods is less than $70 \mathrm{~nm}$ and the rod length is around $200 \mathrm{~nm}$. In Figure 1B, one can clearly see the parallel-lines images, which are ascribed to
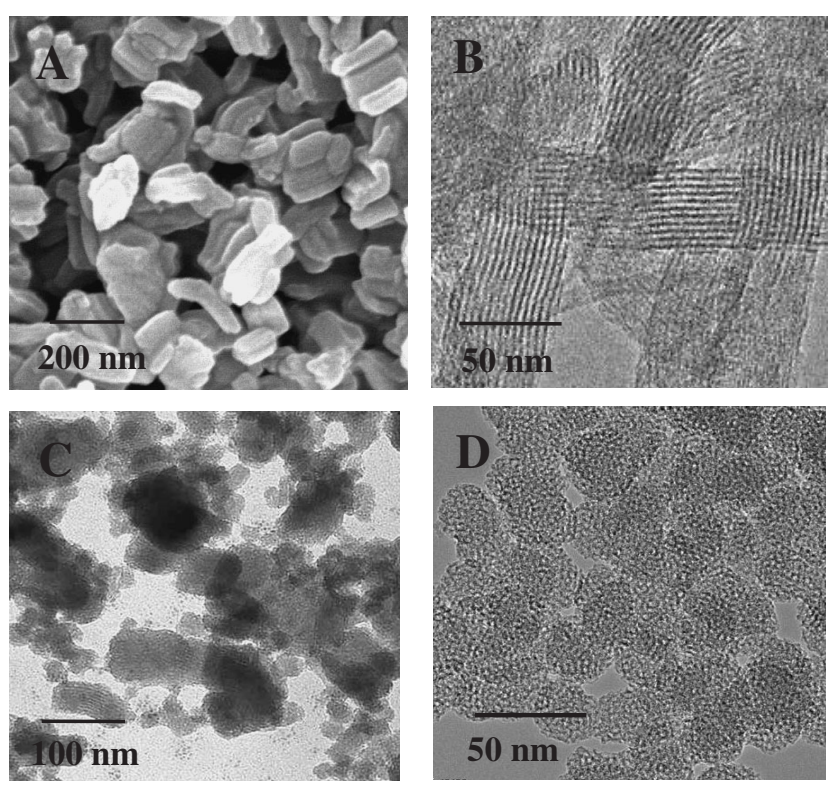

Figure 1. The SEM (A) and TEM (B-D) micrographs of the mesoporous silica synthesized with the $\mathrm{HNO}_{3}$-hydrolyzed TEOS at $30^{\circ} \mathrm{C}$ in the reaction media of different $\mathrm{pH}$ value. (A) SEM and (B) TEM images of silica synthesized at $\mathrm{pH}=9.0 ;$ (C) TEM image of silica synthesized at $\mathrm{pH}=7.0$; (D) TEM image of silica synthesized at $\mathrm{pH}=5.0$.

the well-ordered hexagonal mesostructure in the mesoporous silica nanorods as well as that of the MCM-41 mesoporous silica. It is clear that the nanorods in hierarchical structure are consisted of about tens nanochannels along the rod direction. When the $\mathrm{pH}$ value was decreased to 7.0, the mesoporous silica is a mixture of the nanoparticulate and nanorod, and the particle size is not uniform (Figure 1C). A careful examination reveals that, the orderness of the mesostructure decreases with the particle size. ${ }^{9}$ At $\mathrm{pH}$ value of around 5.0, the morphology completely changes to the fine-particulates with average grain size reduced to nearly $30 \mathrm{~nm}$, and the mesostructure of the nanoparticulates is wormhole-like. To the best of our knowledge, it is the first time to control the morphology and the mesostructures of the mesoporous silica nano-products.

The mesoporous silica nanorods give four well-resolved $\mathrm{X}$ ray diffraction peaks dut to the hexagonal symmetry. When $\mathrm{pH}$ value was decreased to 7.0 or 5.0 , one can find only two broad peaks, which can be assigned to the disordered mesostructure (Figure 2A). Figure 2B shows the representative $\mathrm{N}_{2}$ adsorption-desorption isotherms of the samples in Figure 1. All mesoporous silica samples possess a sharp capillary condensation at relative pressure $P / P_{0}$ of $0.25-0.35$. In addition to the typical mesoporous capillary adsorption, there exists an extra large ad- 

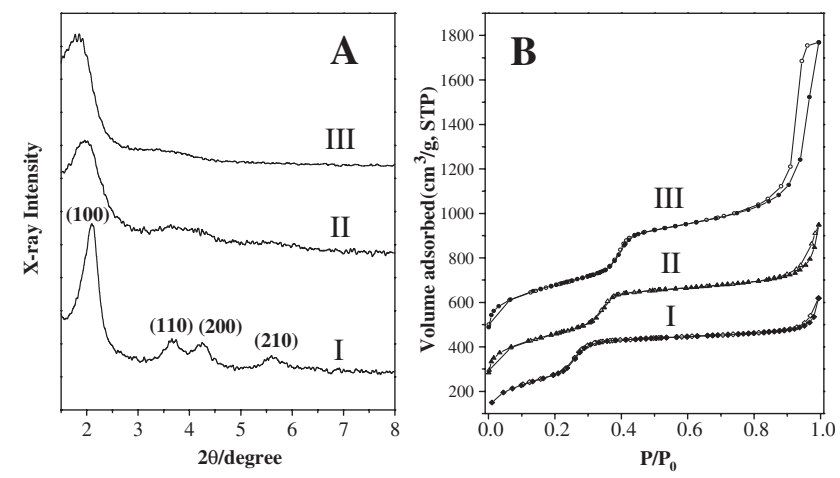

Figure 2. The XRD patterns (A) and $\mathrm{N}_{2}$ adsorption-desorption isotherms (B) of mesoporous silica synthesized with the $\mathrm{HNO}_{3}$-hydrolyzed TEOS at $30^{\circ} \mathrm{C}$ in the reaction media of different $\mathrm{pH}$ value. Sample I prepared at $\mathrm{pH}=9.0$; Sample II prepared at $\mathrm{pH}=7.0$; Sample III prepared at $\mathrm{pH}=5.0$.

sorption at $P / P_{0}>0.85$ in each sample. The appearance of this additional condensation is ascribed to the filling of the textural mesoporosity, which results from the aggregation of the nanoparticles by a capillary force upon drying. ${ }^{9,10}$ As the $\mathrm{pH}$ value decreases, those smaller primary particles intrinsically have the larger adsorption due to the textural porosity. This characteristic adsorption is observed for all samples synthesized from the $\mathrm{HNO}_{3}$-hydrolyzed TEOS between $\mathrm{pH}$ values of 9.0 and 5.0. In contrast, the microsized MCM-41 silica has little or no textural mesoporosity. Table 1 provides the basic physicochemical properties of those mesoporous silica samples. It is clear that all mesoporous silica products possess the advantages of large surface area, high porosity and uniform pore size as the conventional MCM-41. Moreover, one can find that the $V_{\text {tex }} / V_{\text {meso }}$ ratio and pore size increase with the decrease of the $\mathrm{pH}$ value. Because of a higher silica condensation degree occurring at neutral $\mathrm{pH},{ }^{7}$ the mesoporous silicas prepared in a solution of 5.0-7.0, thus, have thicker wall, which leads to a smaller lattice and pore size shrinkage during high-temperature calcination. ${ }^{9}$

Table 1. Physicochemical properties of mesoporous silica ${ }^{a}$ from the $\mathrm{HNO}_{3}$-catalyzed TEOS system of different $\mathrm{pH}$ value at $30^{\circ} \mathrm{C}$

\begin{tabular}{ccccc}
\hline $\begin{array}{c}\mathrm{pH} \text { Value of } \\
\text { the Media }\end{array}$ & $S_{\mathrm{BET}} / \mathrm{m}^{2} / \mathrm{g}$ & $V_{\mathrm{p}} / \mathrm{cm}^{3} / \mathrm{g}$ & $W_{\mathrm{BJH}} / \mathrm{nm}$ & $V_{\text {tex }} / V_{\text {meso }}$ \\
\hline 9.0 & 1165 & 1.06 & 2.3 & 0.72 \\
7.0 & 945 & 1.15 & 2.6 & 1.23 \\
5.0 & 984 & 2.11 & 2.7 & 2.38
\end{tabular}

${ }^{a} S_{\mathrm{BET}}$, the BET specific surface area, $V_{\mathrm{p}}$, total pore volume obtained from $P / P_{0}=0.99 ; W_{\mathrm{BJH}}$, the pore diameter calculated using the BJH method, $V_{\text {meso }}$, framework pore volume obtained from $P / P_{0}=0.50 ; V_{\text {tex }}=V_{\mathrm{p}}-$ $V_{\text {meso. }}$.

Here, we try to explain the basic ideas for controlling the morphology and mesostructural orderness of the mesoporous silica nanoparticles. In a highly acidic medium $(\mathrm{pH}<1.0)$, the hydrolyzed small silica species could attached on the surface of the $\mathrm{C}_{16} \mathrm{TMA}^{+} \mathrm{NO}_{3}{ }^{-}$micelles in water solution via the weak hydrogen-bonding interactions. After a fast-transfer into an alkaline or neutral buffer solution, the interaction between silica species and surfactant was suddenly transformed to the strong electrostatic interaction, which would lead to fast $\mathrm{S}^{+} \mathrm{I}^{-}$selfassembling and silica condensation. Because of such extremely rapid silica condensation on the micelles, the shape of the silica oligomers-surfactant assembles that exist in the acidic media could be preserved. According to the surfactant theory, the $\mathrm{NO}_{3}{ }^{-}$counter ion of $\mathrm{HNO}_{3}$ has strong binding strength to cationic quaternary ammonium surfactant leading to the formation of long cylindrical micelles in the acidic solution. ${ }^{8}$ Consequently, the orderly mesostructured silica nanorods were formed with the $\mathrm{HNO}_{3}$-hydrolyzed TEOS. In contrast, only the silica nanoparticles were formed using the $\mathrm{HCl}$ as acid source, which has a relatively weak binding affinity of the $\mathrm{Cl}^{-}$counter ion toward ammonium quaternary surfactant.

It was aforementioned that a fast silica condensation is intrinsically required to preserve the micellar shape in acidic solution. While the $\mathrm{pH}$ value was decreased to 7.0-5.0, the charge density on the silica species decreases. The lower charge-density silica species has weaker interaction to combine with the ammonium quaternary surfactant that leads to a slower silica condensation onto surfactant micelles. Thus, the longer cylinder micelles would spontaneously break into the shorter cylinders or spheres in the highly diluted buffer solution. Because of the smaller micelle templates and weak surfactant-silica interaction, the particle size and mesostructural orderness, thus, gradually decrease as the $\mathrm{pH}$ value decreases.

On the basis of the concepts of kinetics, there exist two advantages to use a large amount of dilute buffer solution for controlling $\mathrm{pH}$ value. One is that the $\mathrm{pH}$-dependent silica-surfactant assembling and silica condensing reactions occur homogeneously in the buffer aqueous solution that can lead to high homogeneity of the mesoporous silica product. While using the $\mathrm{NaOH}$ solution, a great $\mathrm{pH}$-variation lowers the product homogeneity. Secondly, the concentration of the nanoparticulates or nanorods created is low enough to prevent the further aggregation between nano-products.

In conclusion, we theoretically proposed a convenient method to prepare the mesoporous silica nanorods and nanoparticles in high homogeneity. Different from basic-to-acid quenching process ${ }^{4}$ the acid-to-basic synthetic method can be reasonably applied to incorporate the metal oxide or silane into the silica framework via a cohydrolysis of TEOS and their proper precursors in highly acidic solution. In applications, the metal-oxide incorporated and silane modified mesoporous silica could be used as the high-efficient catalysts and transparent nanofillers in nanohybrids. These subjects will be continued to be developed.

This work is supported by the National Science Council of Taiwan (NSC92-2113-M-006-015).

\section{References}

1 D. R. Rolison, Science, 299, 1698 (2003).

2 T. Yanagisawa, T. Shimizu, K. Kuroda, and C. Kato, Bull. Chem. Soc. Jpn., 63, 988 (1990).

3 C. T. Kresge, M. E. Leonowicz, W. J. Roth, J. C. Vartuli, and J. S. Beck, Nature, 359, 710 (1992).

4 C. E. Fowler, D. Khushalani, B. Lebeau, and S. Mann, Adv. Mater., 13, 649 (2001).

5 S. Shio, A. Kimura, M. Yamaguchi, K. Yoshida, and K. Kuroda, Chem. Commun., 1998, 2461.

6 K. Suzuki, K. Ikai, and H. Imai, J. Am. Chem. Soc., 126, 462 (2004).

7 R. K. Iler, "The Chemistry of Silica: Solubility, Polymerization, Colloid and Surface Properties, and Biochemistry," Wiley, New York (1979).

8 H. P. Lin and C. Y. Mou, Acc. Chem. Res., 35, 927 (2002).

9 H. P. Lin and C. P. Tsai, Chem. Lett., 32, 1092 (2003).

10 T. R. Pauly, Y. Liu, T. J. Pinnavaia, S. J. Billinge, and T. P. Rieker, J. Am. Chem. Soc., 12, 8835 (1999). 